\title{
Plasma Imaging and Optimization of Energy Deposition during Femtosecond-laser Processing
}

\author{
J. Solis, J. Siegel, A. Ferrer and A. Ruiz de la Cruz \\ Instituto de Óptica-CSIC, Serrano 121, 28006-Madrid (SPAIN) \\ j.solis@io.cfmac.csic.es
}

\begin{abstract}
The presentation will provide an overview of our work on plasma imaging techniques for assessing the interaction of fs-laser pulses with dielectrics. Either time-integrated or timeresolved, these techniques can be used in experimental conditions similar to those used during fslaser processing. Their use unveils a number of mechanisms that deteriorate the spatial distribution of energy deposition. These effects can be assessed and minimized leading to highly optimized laser written structures.
\end{abstract}

\section{Introduction}

The advent of non-linear processing of dielectrics using ultrashort laser pulses [1] ant its enormous development over the last years [2] has made necessary the corresponding development of assessment tools aimed either at providing information regarding the dynamics of the process and its fundamental mechanisms or at enabling the optimization of the process in particularly complex interaction scenarios, like those occurring during subsurface processing. In this context, plasma imaging techniques, either time-integrated or time-resolved, provide a unique tool that has been used for long $[3,4]$ for the assessment of ultrafast interactions.

\section{Summary of the presentation}

The presentation will provide an overview of our work regarding the use of plasma imaging for the assessment of the interaction of ultrashort laser pulses with glasses and other dielectrics [5]. These techniques can be adapted in order to reach experimental conditions equivalent to those used during fs-laser processing. Applied to surface processing, time-resolved plasma imaging has the capability to establish a relation between transient local plasma densities and the crater depth or changes in the optical properties of the material [6]. This becomes especially important when searching for an optimum temporal pulse shape for tailored processing [7].

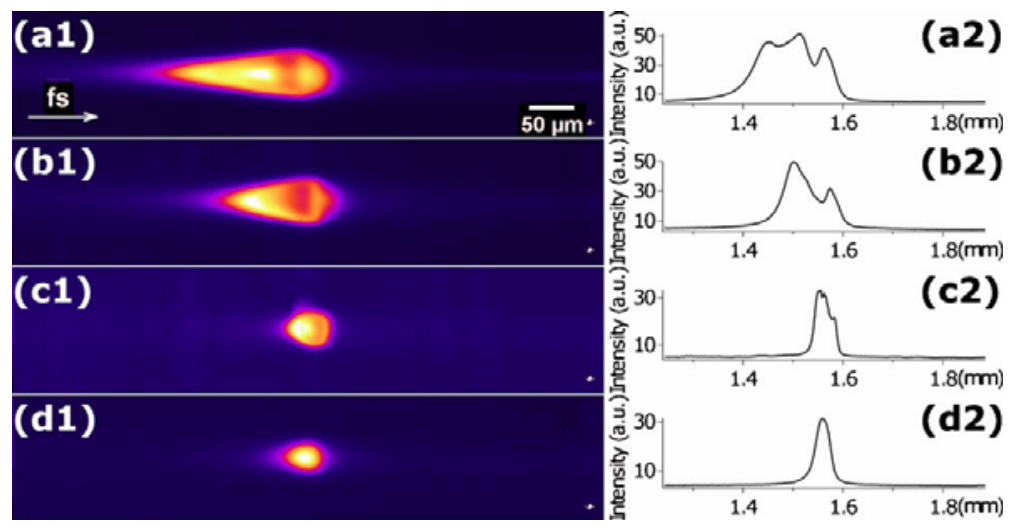

Fig. 1. (Adapted from Ref.[9]) Plasma emission images obtained during subsurface processing for pulses of energies of $9.6 \mu \mathrm{J}$ and pulse durations of (a1) 100, (b1) 250, and (c1) $550 \mathrm{fs}$. In (d1), the pulse duration is $250 \mathrm{fs}$ and the pulse energy is $4 \mu \mathrm{J}$. The corresponding intensity cross sections along the z-axis are shown in the right hand side of the image ((a2),(b2),(c2),(d2))

When applied to bulk processing inside dielectric materials, these techniques prove to be a powerful tool for optimizing the structures produced. Their use in time-resolved configuration [8] has enabled to unveil a number of complex interaction mechanisms including non-linear self-focusing, multiple beam filamentation, and pre-focal energy depletion that act as important energy loss channels that deteriorate the spatial distribution of the deposited 
laser energy. Detailed studies using both time-resolved [8] and time-integrated imaging [9] show how these undesirable effects can be minimized by adjusting the processing parameters. As a consequence, the energy can be deposited much more efficiently and confined in the linear focal volume region, leading to structures with highly optimized performance.

As an illustrative example, Figure 1 shows several plasma emission images (time-integrated) measured upon subsurface irradiation of a commercial Er:Yb-doped phosphate glass for several pulse durations and energies and evidencing the presence of self-focusing, beam filamentation and prefocal depletion effects.

\section{References}

[1] K. M. Davis, K. Miura, N. Sugimoto, and K. Hirao, "Writing waveguides in glass with a femtosecond laser," Optics letters 21, 1729-31 (1996).

[2] See for instace, Femtosecond laser micromachining: photonic and microfluidic devices in transparent materials, R. Osselame, G. Cerullo, R. Raponi, ed., (Springer Verlag, Berlin, 2012).

[3] M. C. Downer, R. L. Fork, and C. V. Shank, "Femtosecond imaging of melting and evaporation at a photoexcited silicon surface," JOSA B 2 , 595-598 (1985).

[4] K. Sokolowski-Tinten, J. Bialkowski, A. Cavalieri, D. von der Linde, a. Oparin, J. Meyer-ter-Vehn, and S. Anisimov, "Transient States of Matter during Short Pulse Laser Ablation," Physical Review Letters 81, 224-227 (1998).

[5] J. Siegel and J. Solis, "Imaging of plasma dynamics for controlled micromachining" in Femtosecond laser micromachining: photonic and microfluidic devices in transparent materials, R. Osselame, G. Cerullo, R. Raponi, ed., (Springer Verlag, Berlin, 2012), Chapter 2.

[6] J. Siegel, D. Puerto, W. Gawelda, G. Bachelier, J. Solis, L. Ehrentraut, and J. Bonse, "Plasma formation and structural modification below the visible ablation threshold in fused silica upon femtosecond laser irradiation," Applied Physics Letters 91, 082902 (2007).

[7] J. Hernandez-Rueda, J. Siegel, D. Puerto, M. Galvan-Sosa, W. Gawelda, and J. Solis, "Ad-hoc design of temporally shaped fs laser pulses based on plasma dynamics for deep ablation in fused silica," Applied Physics A (published online 2012), DOI 10.1007/s00339-012-7238-2.

[8] W. Gawelda, D. Puerto, J. Siegel, a. Ferrer, a. Ruiz de la Cruz, H. Fernández, and J. Solis, "Ultrafast imaging of transient electronic plasmas produced in conditions of femtosecond waveguide writing in dielectrics," Applied Physics Letters 93, 121109 (2008).

[9] A. Ferrer, A. Ruiz de La Cruz, D. Puerto, W. Gawelda, Vallés J. A., M. A. Rebolledo, V. Berdejo, J. Siegel, J. Solis, and A. Ruiz de La Cruz, "In situ assessment and minimization of nonlinear propagation effects for femtosecond-laser waveguide writing in dielectrics," JOSA B 27, 1688-1692 (2010). 